\title{
Growth of Neurospora crassa in Unstirred Liquid Cultures
}

\author{
By O. J. GILLIE* \\ Institute of Animal Genetics, West Mains Road, Edinburgh, 9
}

(Accepted for publication 30 August 1967)

\begin{abstract}
SUMMARY
The growth kinetics of Neurospora in unstirred liquid culture may be accounted for by an exponential and a linear growth phase. If a cubic growth phase exists it is in addition to the exponential and linear phases of growth. A method of measuring the exponential doubling time in unstirred cultures is described which may be usefully applied to fungi which cannot be observed growing exponentially by normal methods. Rate of linear growth is shown to be proportional to the surface area of the culture. Expression of linear growth rates as $\mu \mathrm{g} . / \mathrm{cm} .{ }^{2} / \mathrm{hr}$ would assist in the comparison of data from different laboratories.
\end{abstract}

\section{INTRODUCTION}

Difficulty in analysing the kinetics of growth of filamentous fungi (reviewed by Mandels, 1965) has led to the suggestion that Neurospora (Emerson, 1950) and some other fungi (Machlis, 1957; Marshall \& Alexander, 1960) may have a 'cube-root' growth phase corresponding to the logarithmic growth phase of other organisms. During the cube-root phase the mycelium is considered to be a sphere with radius increasing at constant rate, such that the cube root of the mycelial mass would increase in direct proportion to time. Other workers have found clear evidence for an exponential growth phase in fungi (Zalokar, 1959; Pirt \& Callow, 1960) but this does not preclude the existence of a cubic growth phase under other conditions. A theoretical discussion of cubic growth has been made by Pirt (I966).

Experiments described in the present paper show that the growth of Neurospora in unstirred cultures may be analysed into an exponential phase followed by a linear phase. No evidence was found to suggest the existence of a cubic phase of growth; experiments of Emerson (I950) may be reinterpreted to support this conclusion.

\section{METHODS}

Except where stated, all experiments were made with the St Lawrence wild-type strain STA of Neurospora crassa grown at $25^{\circ}$ on Vogel liquid minimal medium (Vogel, 1956) with $2 \%(\mathrm{w} / \mathrm{v})$ sucrose in unstirred bottles or beakers. The bottles used were $12 \mathrm{oz}$ medical flats containing $100 \mathrm{ml}$. medium and plugged with cotton-wool. These bottles were sloped at about $10^{\circ}$ to the horizontal. Beakers of known diameter were filled with medium to a depth of I cm. and covered with two layers of cheesecloth stuck down with autoclavable adhesive tape. The cheesecloth was covered with a sheet of aluminium foil and the beakers were then autoclaved. The aluminium foil was removed and discarded at the time of inoculation. Growth was estimated as dry weight of mycelial pads (collected by filtration through paper on a Büchner funnel) after freeze-drying.

*Present address: National Institute for Medical Research, The Ridgeway, Mill Hill, London, N.W.7 
Inocula consisted of macroconidia counted in a haemocytometer and diluted in distilled water. Cultures were inoculated together by using one inoculum or a series of diluted inocula. Duplicate cultures were harvested at suitable times.

\section{RESULTS}

Initial observations of Neurospora crassa wild-type strain STA growing in unstirred vessels showed that there was a phase of accelerating growth, followed by a linear phase of growth. The growth rate in the linear phase varied with the type of vessel in which growth occurred, e.g. whether beakers or bottles. Furthermore, the onset of linear growth was delayed when the inoculum was diluted, regardless of the type of vessel used. Results showing these effects are given in Fig. I. These observations led to experiments specifically designed to investigate the effect of surface area and inoculum size on growth in liquid medium.

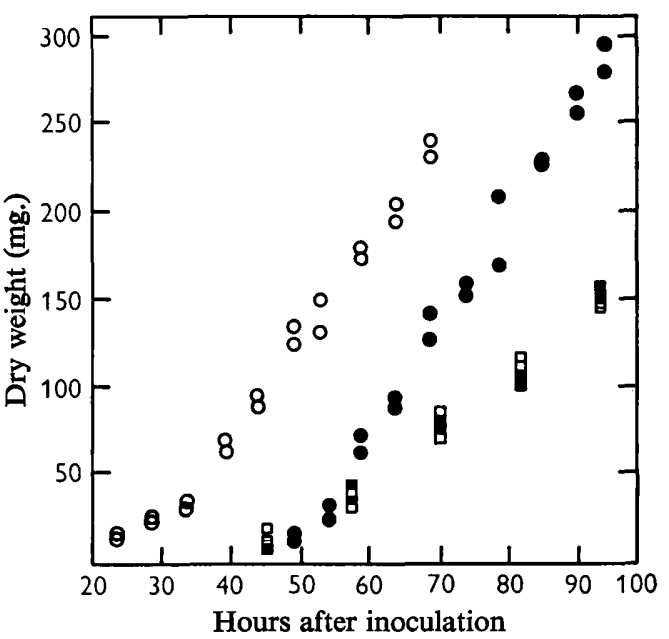

Fig. I

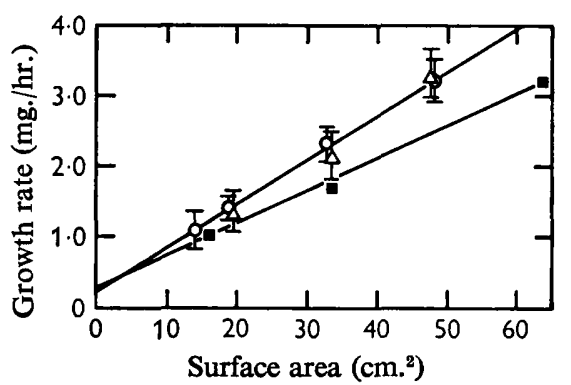

Fig. 2

Fig. I. Growth of Neurospora crassa wild-type strain STA in various circumstances. $\bigcirc$, growth in bottles with an inoculum of $2.6 \times 10^{6}$ conidia per bottle; $\theta$, growth under the same conditions with an inoculum of $2.6 \times 10^{8}$ conidia per bottle; $\square$, growth in $500 \mathrm{ml}$. beakers containing $135 \mathrm{ml}$. of medium with an inoculum of $1.5 \times 10^{8}$ conidia; $\mathbf{m}$, growth under the same conditions except that the beakers contained only $90 \mathrm{ml}$. medium. Surface area of growth in the bottles was about $80 \mathrm{~cm} .^{2}$ and in the beakers $49 \mathrm{~cm} .^{2}$. The data for growth in bottles has been further analysed in Table $\mathbf{I}$.

Fig. 2. Growth of Neurospora crassa wild-type strain STA in beakers of various diameters. Linear growth rate is plotted against surface area of the culture for growth of strain STA in beakers of various diameters. The linear growth rate is calculated from raw data of the type shown in Fig. I, as a regression of mycelial weight against time. The $95 \%$ confidence interval was calculated from the standard error using Student's $t$ distribution. Data from two separate experiments are plotted as circles and triangles with confidence intervals indicated by vertical lines. $\mathbf{n}$, data taken from Emerson (1950). (See text for explanation.)

\section{Linear growth and the surface area of the culture}

Beakers of different sizes were used to vary the surface area of the cultures while the inoculum size was kept constant. Linear growth rates in beakers were measured in two experiments summarized in Fig. 2, which shows that the linear growth rates observed 
were directly proportional to the surface area of the culture. Linear growth rate may then be expressed as $6 \mathrm{r} \mu \mathrm{g} . / \mathrm{cm} .{ }^{2} / \mathrm{hr}$ for this strain, sTA, a measure which should be largely independent of the type of vessel used for cultivation.

Emerson's original experiments purporting to show a cubic growth phase in Neurospora were also made in vessels of different diameters although no explanation of this is given in his paper. The last three growth points of each of his curves fall on a straight line and may be equated with the linear phase of growth identified here. The linear growth rates taken from Emerson's data in this way also show a direct relationship to surface area of the culture (see Fig. 2) and may be used to calculate an overall linear growth rate of $46 \mu \mathrm{g} . / \mathrm{cm} .^{2} / \mathrm{hr}$ for his strain.

\section{The phase of increasing growth rate}

Preceding the linear phase of growth in unstirred cultures there was a phase during which the growth rate increased until it reached the constant linear rate (Fig. I). Logarithmic plots of this early phase of increasing growth rate give increasing doubling times of 2-5 hr or more. It seems likely that this phase of increasing growth rate is the last part of the logarithmic phase, during which growth is decelerating, before growth rate becomes constant and doubling time ceases to have any meaning. Zalokar (1959) showed, by measuring protein increase after extraction, that germination of conidia was followed by a period of exponential growth with a doubling time of about $2 \mathrm{hr}$, thus showing that initial growth of an inoculum was exponential. Further indirect evidence for the early exponential growth of inocula is considered below.

\section{The relationship between inoculum size and growth}

Theoretical. The relationship between inoculum size and the mass of a culture which is growing exponentially may be deduced from the well-known exponential growth equation $d M / d t=\mu M$, where $M=$ mass of the culture at time $t$ and $\mu$ is a constant known as the 'specific growth rate'. This equation may be integrated for initial conditions where $M$ is the mass of the culture at time $t$, and $M_{0}$ the mass of the culture at $t=0: \log _{e} M-\log _{e} M_{0}=\mu t$. In this equation $M_{0}$ is usually considered to be constant and $M$ to be variable. We may consider the case where $M_{0}$ (the inoculum size) is varied and $M$ is chosen as an arbitrary constant. Then we find that $-\log _{e} \mathrm{M}_{0}$ is proportional to $t$, i.e. in a series of experiments with exponentially growing cultures started from inocula of different sizes the time taken to reach an arbitrary constant mass will be proportional to the logarithm of the inoculum size. If $M_{0}$ is plotted against $t$ in such a series of experiments, then the slope will be $\mu$, the specific growthrate constant. The doubling time, $t_{d}$, may be readily calculated since

$$
t_{d}=\frac{\log _{e} 2}{\mu} .
$$

If only two inocula, $M_{1}$ and $M_{2}$, are being considered where we choose $M_{1}=$ Io. $M_{2}$ then

$$
t_{d}=\frac{\log _{e} 2\left(\mathrm{t}_{2}-t_{1}\right)}{\log _{e} \mathrm{IO}},
$$

where inoculum $M_{1}$ reaches constant mass $M_{c}$ at $t_{1}$ and inoculum $M_{2}$ reaches constant mass $M_{c}$ at $t_{2}$. The value $\left(t_{2}-t_{1}\right)$ is called the 'unit time difference' for inoculum dilution of $10^{-1}$. 
The expressions derived above are only formally true for exponentially growing cultures, but they may also be applicable to cultures which have an exponential growth phase which gradually changes into a linear growth phase, as seems to be found during the growth of Neurospora in unstirred cultures. Indeed, when a constant figure for the doubling time (calculated from inoculum dilution experiments) is obtained under various conditions, then this may be taken to indicate that an early exponential phase of growth exists. If there were a cubic growth phase, then we might expect $-M_{0}^{\frac{1}{3}} \alpha t$, such that $M_{0}$ (inoculum size) is a cubic rather than a logarithmic function of time.

Table I. Neurospora crassa wild-type strains STA and EMA. The relationship of growth rate, and time difference, to inoculum dilution for growth in unstirred cultures

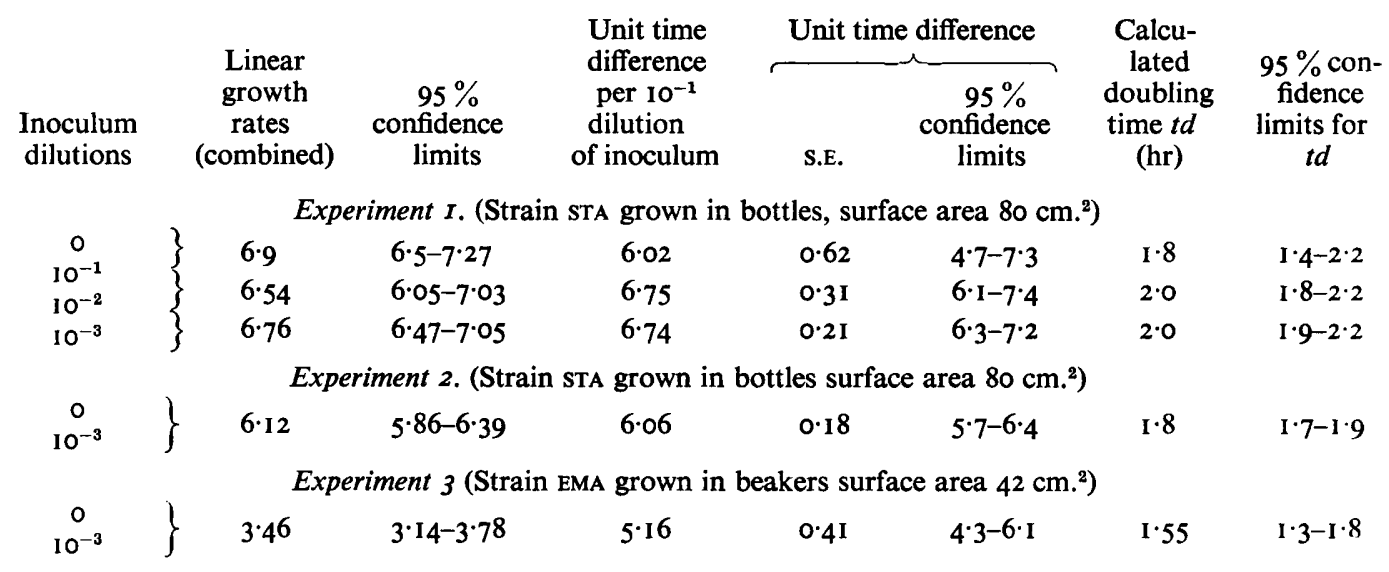

An undiluted inoculum of about $10^{6}$ conidia per culture was used in all experiments. The raw data used in Expt. 2 is depicted in Fig. $\mathbf{I}$.

Results. Table I shows the analysis of results of growth experiments in which the inoculum size was varied. Measurements of rate of growth during the linear phase were made and were found not to vary significantly $(P>0.01)$ at different inoculum dilutions. The calculated regressions were used to calculate the 'unit time difference' for a standard inoculum dilution of $10^{-1}$. This is the time difference in hours taken between successive inoculum dilutions of $\mathrm{IO}^{-1}$ to reach any given weight, and may be used to calculate the doubling time as shown above.Three independent estimates of the unit time differences in experiments with Neurospora crassa wild-type strain STA (Expts. I and 2, Table I) were homogeneous $\left(\chi^{2}, P=0.5^{-0.7}\right)$, whereas the unit time differences for wild-type strain EMA possibly differed from those for strain STA $\left(\chi^{2}\right.$, $P=0.0 \mathrm{I}-0.05)$. Calculation of the doubling time on the basis of these figures, pooled data for Expts. I and 2, gives a value of I.84 hr for strain STA ( $95 \%$ confidence limits $\mathrm{I} \cdot 74-\mathrm{I} \cdot 94 \mathrm{hr}$ ). These values are in reasonable agreement with doubling times for strain STA in shaken logarithmic cultures in the same medium at $25^{\circ}$, of $2 \cdot 3 \mathrm{hr}(95 \%$ confidence limits $\mathrm{I} \cdot 7-2 \cdot 9 \mathrm{hr}$ : C. F. Curtis, personal communication).

The unit time differences would be expected to decrease by a factor of two for each tenfold dilution of inoculum if growth were 'cubic'. They did not, however, vary significantly with dilution and in fact were entirely consistent with exponential growth and difficult to account for on any other basis. 


\section{DISCUSSION}

The results presented support the conclusion that the early growth of Neurospora crassa wild-type strains in unstirred liquid cultures is exponential and that the exponential growth rate slowly declines until growth becomes linear. The change from exponential to linear growth seems to occur at about the time when the mycelial mass becomes established on the surface of the liquid. At this stage nutrients are still in plentiful supply, since the mass will increase more than tenfold by subsequent linear growth. The rate of linear growth is proportional to the surface area of the culture; this may be interpreted as resulting from the limited rate of diffusion of air or nutrients into the mycelial mat. In fact the mould is growing at a nutrient/air interface and does not grow downwards into the liquid medium probably because of lack of oxygen below the surface. Linear growth may also, however, be obtained in stirred and aerated cultures in which the mycelium occurs in small pieces. In such cultures the constant but limiting supply of air must be responsible for the constant linear growth rate.

The linear growth rate found during growth in beakers was not increased by pumping in sterile air, suggesting that it is the rate of diffusion of air into the mycelial mat rather than into the beaker which was limiting. These results are in agreement with the conclusion of Pirt (I966), based on theoretical considerations of a comparable situation, that the growth-limiting nutrient will almost inevitably be oxygen when fungi grow as spherical pellets in submerged culture.

The results described here throw some doubt on whether a cube-root growth phase exists for Neurospora crassa. Although all observations can be accounted for in terms of a linear and exponential growth phase, a cubic growth phase may occur and not be detectable by present methods. However, if a cubic growth phase occurred in addition to an exponential growth phase, it would not be expected that an inoculum dilution experiment of the type described above would give results entirely consistent with exponential growth. It should be pointed out that the evidence of Marshall \& Alexander (1960) for a cubic growth phase in fungi and actinomycetes is indirect, depending upon measurements of rate of oxygen uptake. It is difficult to exclude the possibility that such measurements might be affected in a systematic way by changes in metabolic control systems which were growth-dependent.

I should like to thank Miss C. Stake and Mrs K. Henderson for excellent technical assistance, and Miss J. Skegg for expert statistical assistance.

\section{REFERENCES}

EMERSON, S. (1950). The growth phase in Neurospora corresponding to the logarithmic phase in unicellular organisms. J. Bact. 6o, $22 \mathrm{I}$.

MACHLIS, L. (1957). Factors affecting the log phase of growth of the filamentous fungus, Allomyces macrogynus. Am. J. Bot. 44, 113.

Mandels, G. R. (1965). Kinetics of fungal growth. In The Fungi. An Advanced Treatise. Ed. by G. C. Harrison \& A. S. Sussman, vol. I. New York and London: Academic Press.

Marshall, K. C. \& Alexander, M. (1960). Growth characteristics of fungi and actinomycetes. J. Bact. 80, 4I 2 . 
I84

O. J. GILLIE

PIRT, S. J. (1966). A theory of the mode of growth of fungi in the form of pellets in submerged culture. Proc. $R$. Soc. B I66, 369.

PIRT, S. J. \& Callow, D. S. (1960). Studies of the growth of Penicillium chrysogenum in continuous flow culture with reference to penicillin production. J. appl. Bact. 23, 87 .

VoGEL, H. J. (1956). A convenient growth medium for Neurospora (medium N). Microb. Genet. Bull. $\mathbf{r}, 42$.

ZAloKAR, M. (1959). Enzyme activity and cell differentiation in Neurospora crassa. Am. J. Bot. 46, 555 . 